\title{
VIII Nuclear Physics in Astrophysics International Conference (NPA8): Preface
}

\author{
Marco La Cognata ${ }^{1}$, Marcello Lattuada ${ }^{2,1}$, Sara Palmerini ${ }^{3,4}$, Rosario Gianluca Pizzone ${ }^{1}$, and \\ Claudio Spitaleri ${ }^{2,1}$ \\ ${ }^{1}$ INFN - Laboratori Nazionali del Sud, Via S. Sofia 62, 95123 Catania, Italy \\ ${ }^{2}$ Dipartimento di Fisica e Astronomia, University of Catania, Via S. Sofia 64, 95123 Catania, Italy \\ ${ }^{3}$ Dipartimento di Fisica e Geologia, University of Perugia, Via A. Pascoli, 06123 Perugia, Italy \\ ${ }^{4}$ INFN - Sezione di Perugia, Via A. Pascoli, 06123 Perugia, Italy
}

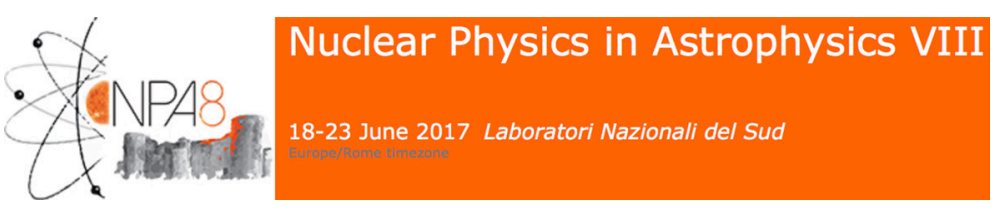

NPA8 is the successor of earlier events held in Eilat, Israel, in 2001 and, with a 2-year frequency, in Debrecen (Hungary, 2 times), in Frascati (Italy), Eilat (Israel) and in York (UK). In the same spirit as the earlier editions, NPA8 was the showcase for the most recent developments in the field of nuclear astrophysics covering a wide range of topics from fundamental aspects to instrumentation and astrophysical applications. Room was given not only to the traditional aspects of experimental nuclear astrophysics, such as direct measurements above and under ground and indirect approaches, but also to emerging fields such as the measurement of fusion reactions in laser induced plasmas and of photodissociation reactions. Also significant room has been given to novel experimental techniques that might have important impact on future nuclear astrophysics experiments and on astrophysical modelling and observations, which constitute the necessary motivations and background of the proposed measurements. The event was attended by a large number of researchers at the start of their scientific career together with well-established senior scientists. Such a diversity in experience, nationality and research expertise made NPA8 an ideal platform for cross fertilisation between the disciplines, stimulating new ideas and scientific networks.

During NPA8 a special event was organised, to celebrate the scientific achievements of the conference chairman, C. Spitaleri, on the occasion of his retirement. 


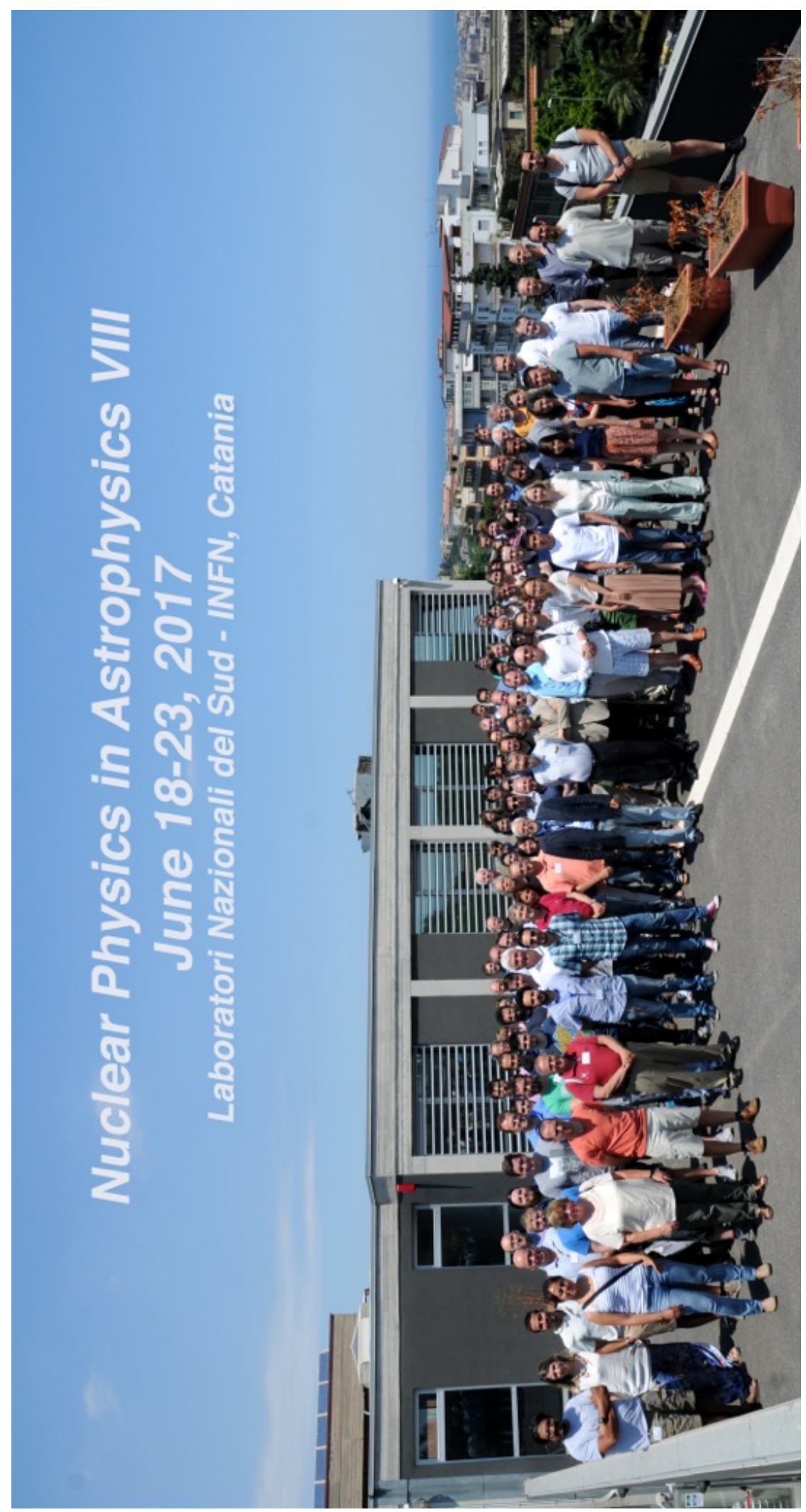

